\title{
TIME IN DYNAMICAL SYSTEMS
}

MAURICE COURBAGE

Received 5 March 2004

We review some ideas and concepts on the irreversibility of deterministic dynamical systems that have been discussed during several years of collaboration with Ilya Prigogine and B. Misra.

\section{Introduction}

The irreversibility problem has an outstanding status in science. The strong debates raised by the dichotomy of the reversible microscopic dynamics and the irreversible macroscopic physics have not succeeded to solve definitively the main important conceptual problems. They are nowadays so open that, according to Uhlenbeck, "even their formulation is often still controversial." Many physicists believe that there is a generally admitted solution. We may be persuaded by the opposite if we read the debate between Pierels and Uhlenbeck [15, page 511].

A definition of "good" nonequilibrium statistical Gibbs ensemble and a dynamical system theory of the approach to equilibrium with an entropy increase law are still lacking. The kinetic theory, even in moderately dense fluids along the Boltzmann-type equation, faces many open problems [2]. On the other hand, the stochastic models, like the urn Ehrenfest model, solved by Kac [8], provide best models of the monotonic approach to equilibrium. They result from phenomenological Chapman-Kolmogorov equation and Markovian assumption (or more generally, from the property of Markov semigroup of operators). The Kac ring model [8] illustrates the nature of the probabilistic assumptions added to deterministic dynamics in order to obtain the Markovian description. Until now, the "standard" derivations of a time monotonic increase of entropy from the Liouville equation come through Markovian limits assumptions (many references contain such methods, among them are the books of Résibois and De Leener [11], and Spohn [14]).

The Gibbs ideas on the approach to equilibrium as a mixing dynamical system gave new impetus to Ergodic theory. Gibbs promoted a new statistical theory of the approach to equilibrium in terms of nonstationary ensembles (i.e., probability distributions on the 
phase space $\Gamma$ of the macroscopic system) which can be summarized as follows. Let $S_{t}$ be a smooth transformation on a manifold $\Gamma$ (i.e., the Hamiltonian flow on the phase space of the energy surface) and let $\mu$ be an invariant probability measure on the phase space (i.e., the equilibrium microcanonical ensemble). Out of equilibrium, the system is described by a probability measure $\nu_{0}=v$ which is noninvariant under the flow $S_{t}$ and given at time $t$ by

$$
\nu_{t}(A)=\nu\left(S_{-t} A\right)
$$

for any measurable subset $A$ of $\Gamma$. Let $U_{t}$ be the group of operators defined by

$$
U_{t} \nu(A)=\nu\left(S_{-t} A\right) \text {. }
$$

If $\nu$ has square integrable density, then $U_{t}$ reduces to the Koopman operator on $L^{2}(\Gamma, d \mu)$ given by

$$
U_{t} \rho(x)=\rho\left(S_{-t}(x)\right),
$$

which transforms a probability density into a probability density. The system will approach equilibrium if for any typical initial noninvariant distribution $\nu, \nu_{t}$ tends to $\mu$ as $t \rightarrow \infty$. There are many ways to define this convergence. One of them is suitable to the case of compact phase space and is defined by

$$
\int_{\Gamma} \varphi(x) d \nu_{t}(x) \longrightarrow \int_{\Gamma} \varphi(x) d \mu(x)
$$

as $t \rightarrow \infty$, for all continuous functions $\varphi$ on $\Gamma$. This is sufficient to our presentation. According to Gibbs, the shape of any subset $A$ will change boldly under the action of the flow, although conserving a constant volume $\mu(A)$, winding as a twisted filament filling $\Gamma$ in such a way that its fraction in any subset $B$ will be equal to the fraction of $A$ in $\Gamma$ :

$$
\mu\left(S_{-t} A \cap B\right) \longrightarrow \mu(A) \mu(B)
$$

for $t \rightarrow \infty$. This mixing property implies the approach to equilibrium for any distributions $\nu$ having a density $\rho$ with respect to $\mu$.

The problem of irreversibility has been raised by Loschmidt and has to be distinguished from the problem of the approach to equilibrium. It is formulated in the following terms. The Hamiltonian dynamics admits a time inversion operation (i.e., the velocity inversion) which is a one-to-one $\mu$-preserving transformation $I$ such that

(i) $I^{2}=1$, that is, $I=I^{-1}$,

(ii) $I S_{t} I=\left(S_{t}\right)^{-1}$.

This operation allows the system to go back in time whatever the initial condition is. It induces a transformation onto the probability distributions $\nu \rightarrow V \nu$ defined by

$$
V v(A)=v(I A)
$$

Definition 1.1. The intrinsic irreversibility of a dynamical system means that there exists a class $\mathfrak{C}^{+}$of initial distributions that tend to equilibrium for $t \rightarrow+\infty$ but not for $t \rightarrow-\infty$, 
that is, $\mathfrak{C}^{+} \cap V \mathfrak{C}^{+}=\phi$. Moreover, there exists a monotonically increasing entropy $\mathbf{S}\left(v_{t}\right)$ which tends, as $t \rightarrow+\infty$, to its maximum, reached only at equilibrium.

The above condition means that the set of all initial distributions that converge to equilibrium, denoted by $\mathfrak{D}^{+}$, is not invariant under the time reversal $V$, for it includes the asymmetric subset $\mathfrak{C}^{+}$.

The class of the dynamical systems for which this programm can be realized is not empty. How big is it? This is still an unsolved difficult problem. Here we explain how it was considered for the Hyperbolic and $K$-systems.

\section{Irreversibility in hyperbolic and $K$-systems}

The Lorentz gas in two dimensions is a system of noninteracting particles moving with constant velocity and being elastically reflected from periodically distributed scatterers. The scatterers are supposed to be fixed disks. Each particle of the billiard is described as follows. Let $Q$ denote the subset of $\mathbb{R}^{2}$ of all disjoint disks; the one particle phase space is the set of $(q, v) \in\left(\mathbb{R}^{2}-Q\right) \times S^{1}$ with the Lebesgue measure $\lambda_{Q}$. A special representation of the flow (a Poincare section) is given by the successive colliding arrows, where a colliding arrow represents the state of the colliding particle on the obstacle, $x=(r, \theta)$, $r$ being the curvilinear abscissa of its impact point on the disk, and $\theta$ its impact angle with the outer normal. Sinar [12] has shown that the mapping $T$, which associates to each colliding arrow $x=(r, \theta)$ the next one $T x$, is a hyperbolic $K$-system with respect to the invariant Lebesgue measure (Gallavotti and Ornstein [6] proved that the system is a Bernoulli scheme). This implies mixing and the approach to equilibrium. It is possible to find the intrinsic irreversibility property as resulting from its hyperbolic structure.

We recall some basic definitions on hyperbolic systems.

The uniformly hyperbolic (Anosov) systems have a geometrical structure similar to the ergodic automorphism of the torus $\mathbb{T}^{2}$ (the famous Arnold "cat map"). Let $S$ be a smooth transformation on a manifold $\Gamma$ such that for each point $x \in \Gamma$, there is a splitting of the tangent space $T_{x} \Gamma$ into two linear subspaces $E_{x}^{s}$ and $E_{x}^{u n}$ :

$$
T_{x} \Gamma=E_{x}^{s}+E_{x}^{u n}, \quad E_{x}^{s}+E_{x}^{u n}=m .
$$

Let $D_{x} S$ denote the derivative of $S$ at point $x$. The above splitting is invariant in the following sense:

$$
E_{S x}^{s}=\left(D_{x} S\right) E_{x}^{s}, \quad E_{S x}^{u n}=D_{x} S\left(E_{x}^{s}\right)
$$

The phase space of a hyperbolic system is decomposed into a family of contracting manifolds $\Sigma_{s}(x)$ :

$$
\Sigma_{s}(x)=\left\{y \mid d\left(S_{t} y, S_{t} x\right) \leq c \lambda^{t} d(y, x)\right\}
$$

Here $S_{t}$ denotes the $t$ th power of $S$. Dilating manifolds $\Sigma_{u n}(x)$ can be defined similarly. Smooth pieces of these manifolds yield two partitions of $\Gamma$ denoted by $\xi_{c}$ and $\xi_{d}$, respectively. The system is said to be reversible when there is a one-to-one transformation $I$ as 
above. We will consider the case where I maps contracting fibers onto dilating fibers and conversely.

Ilya Prigogine always believed in the universality of the irreversible processes and in the fact that their origin is to be found only in some universal properties of the deterministic dynamics. In the sixties, Prigogine and his school proposed a general criterion on the dynamical systems for which an irreversible kinetic equation can be derived from the Liouville equation of the nonequilibrium probability distributions. This criterion called "the dissipativity condition" was formulated in terms of the existence of some nonvanishing "collision operator." This expresses in his spirit the intrinsic irreversibility of these dynamical systems. With an outstanding group of researchers around him, they went to propose a general theory of irreversible kinetic equation which is "equivalent" to the Liouville equation. The derivation of this equivalence was so tedious that many physicists found it too hard to follow. Grécos and others, among them the author, made some contributions to clarify the above condition. In 1978, Misra found a Lyapounov function for the Liouville equation of the $K$-systems. Then started the author's collaboration with him. The spectral analysis of the time operator in the Baker transform led to surprising results. Prigogine was very enthusiastic to discover that the "Ergodic theory" which he criticized in the seventies, as insufficient to explain the irreversible evolution to equilibrium, is able to illustrate so nicely the above equivalence. In a joint work with him and Misra [10], we obtained the above equivalence for the baker transformation, which has been extended successively to all Bernoulli schemes [4] and to all $K$-systems [7]. The main idea was to construct a positivity-preserving nonunitary transformation $\Lambda$ acting on $L^{2}(\Gamma, d \mu)$ which transforms the probability densities into probability densities and such that

$$
\Lambda U_{t}=W_{t} \Lambda
$$

for any $t \geq 0$, where $W_{t}$ is a semigroup of Markov operators. This property was called "intrinsic randomness of the dynamical system."

In a joint work with Ilya Prigogine, we have shown that the hyperbolic dynamical systems provide a new model to find the intrinsic irreversibility $[3,5]$ in terms of instability. The class $\mathfrak{C}^{+}$of initial distributions that tend to equilibrium for $t \rightarrow+\infty$ but not for $t \rightarrow-\infty$ are those (singular) probability measures that are concentrated on the dilating fibers such that their conditional measures on these fibers are absolutely continuous with respect to the invariant measure $\mu$. Then, the transformation $\Lambda$ can be defined also for this class in such a way that the above equivalence holds. The transformed distribution has a probability density $\tilde{\rho}_{t}$ with monotonically increasing entropy

$$
\mathbf{S}\left(v_{t}\right)=-\int \tilde{\rho}_{t}(x) \log \tilde{\rho}_{t}(x) d \mu(x)
$$

The maximum is only reached at equilibrium.

The discrimination between future-oriented admissible initial distributions (smooth measures supported by dilating fibers) and their time reversed (smooth measures supported by the contracting fibers) can be quantified by their entropy content. This entropy is finite only for the first family. The dynamics acting on these distributions in future will 
converge to equilibrium. Some physical and probabilistic interpretations of the $\Lambda$ transformation have been discussed in, for example, $[1,9]$.

We briefly illustrate the exclusion of the singular measures supported by contracting fibers in the Sinal billiard. A local dilating fiber passing through a point $x=(r, \theta)$ corresponds to an ensemble of initial conditions of particles which, after executing an infinite number of collisions, will form focusing beam around the trajectory of $x$. On the other hand, a local contracting fiber passing through $x$ corresponds to an ensemble of initial conditions of particles that were in the remote past a focusing beam around $x$. It is clear that this dichotomy corresponds to the macroscopically observed time symmetry breaking.

\section{Concluding remarks}

The above appealing scheme has two limitations. Although the equivalence theory is possible for all $K$-systems, it is not always meaningful as it follows from the fact that an ideal gas has also a $K$-system property. Recently, with Kaminski, we introduced a concept of space-time intrinsic randomness so that it was possible to show that in extended spacetime dynamical systems $Z^{d}$-actions, $K$-property is a necessary and sufficient condition of intrinsic randomness. This leads to a discrimination between the Lorentz gas which is space-time intrinsically random and the ideal gas which is not. It is to be noted that the above equivalence is not unique. Recently, Sinaî gave another method to find an $\mathrm{H}$ theorem in hyperbolic dynamical systems [13]. The second limitation concerns the rather restricted class of $K$-systems. $K$-property is not expected to hold in the more interesting Hamiltonian systems and in this case, the problems of the approach to equilibrium and irreversibility are still largely open.

Nevertheless, the many unanswered questions as to applications of the above scheme deserve more investigations at least in the field of hard spheres which have undergone during the last decade crucial progress. Until the last period of his life, Ilya Prigogine continued to believe in the possibility of finding a general semigroup of evolution from Liouville equation. In my opinion, his support to all efforts in the field of irreversible processes was very important and he will continue to be for me and for many others our wonderful teacher.

\section{References}

[1] I. Assani and M. Courbage, On the loss of information in the transition from deterministic systems to probabilistic processes, Lett. Math. Phys. 12 (1986), no. 4, 257-265.

[2] E. G. D. Cohen, The kinetic theory of fluids — an introduction, Phys. Today (1983), 64-73.

[3] M. Courbage, Intrinsic irreversibility of Kolmogorov dynamical systems, Phys. A 122 (1983), no. 3, 459-482.

[4] M. Courbage and B. Misra, On the equivalence between Bernoulli dynamical systems and stochastic Markov processes, Phys. A 104 (1980), no. 3, 359-377.

[5] M. Courbage and I. Prigogine, Intrinsic randomness and intrinsic irreversibility in classical dynamical systems, Proc. Natl. Acad. Sci. USA 80 (1983), no. 8, 2412-2416.

[6] G. Gallavotti and D. S. Ornstein, Billiards and Bernoulli schemes, Comm. Math. Phys. 38 (1974), 83-101 . 


\section{Time in dynamical systems}

[7] S. Goldstein, B. Misra, and M. Courbage, On intrinsic randomness of dynamical systems, J. Statist. Phys. 25 (1981), no. 1, 111-126.

[8] M. Kac, Probability and Related Topics in Physical Sciences, Lectures in Applied Mathematics, vol. 1, Interscience Publishers, New York, 1959.

[9] S. Martinez and E. Tirapegui, A possible physical interpretation of the $\Lambda$ operator in the Prigogine theory of irreversibility, Phys. Lett. A 110 (1985), no. 2, 81-83.

[10] B. Misra, I. Prigogine, and M. Courbage, From deterministic dynamics to probabilistic descriptions, Phys. A 98 (1979), no. 1-2, 1-26.

[11] P. Résibois and M. De Leener, Classical Kinetic Theory of Fluids, John Wiley \& Sons, New York, 1977.

[12] Ya. G. Sinắ, Dynamical systems with elastic reflections, Soviet Math. Dokl. 4 (1963), translated in Russian Math. Surveys 25 (1970), no. 2, 137-189 (Russian).

[13] - Topics in Ergodic Theory, Princeton Mathematical Series, vol. 44, Princeton University Press, New Jersey, 1994.

[14] H. Spohn, Large Scale Dynamics of Interacting Particles, Texts and Monographs in Physics, Springer-Verlag, Berlin, 1991.

[15] G. E. Uhlenbeck, Problems of statistical physics, The Physicist's Conception of Nature (J. Mehra, ed.), D. Reidel Publishing, Dordrecht, 1973.

Maurice Courbage: Laboratoire de Physique Théorique de la Matière Condensée, Fédération Matière et Systèmes Complexes, Université Paris 7 - Denis Diderot, 75251 Paris Cedex 05, France

E-mail address: courbage@ccr.jussieu.fr 


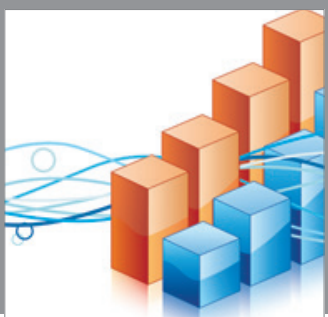

Advances in

Operations Research

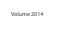

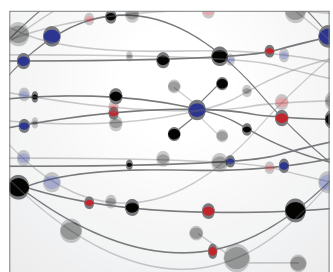

\section{The Scientific} World Journal
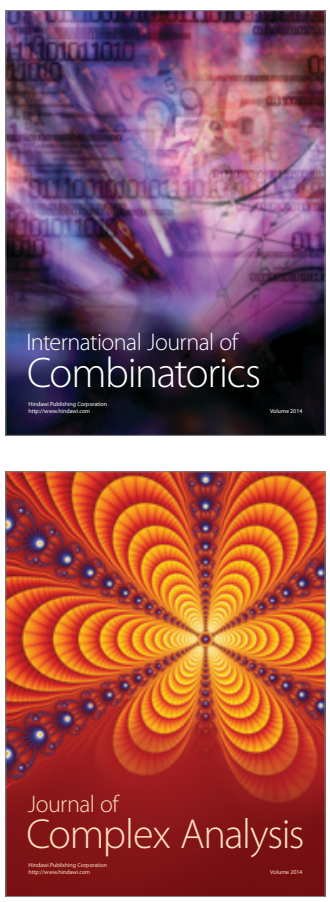

International Journal of

Mathematics and

Mathematical

Sciences
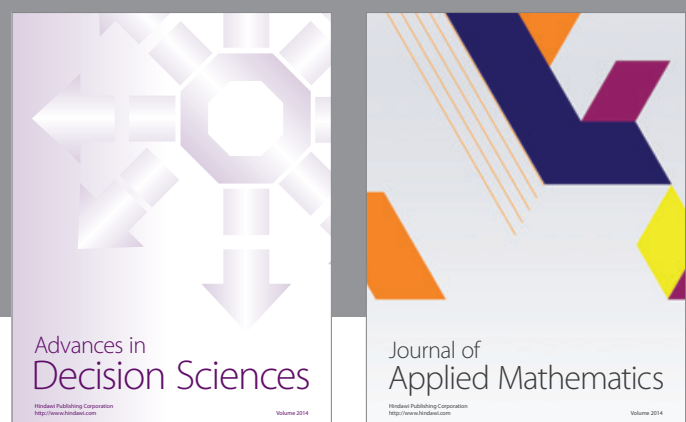

Journal of

Applied Mathematics
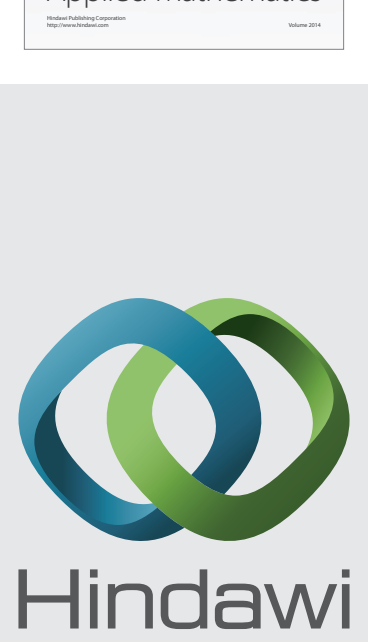

Submit your manuscripts at http://www.hindawi.com
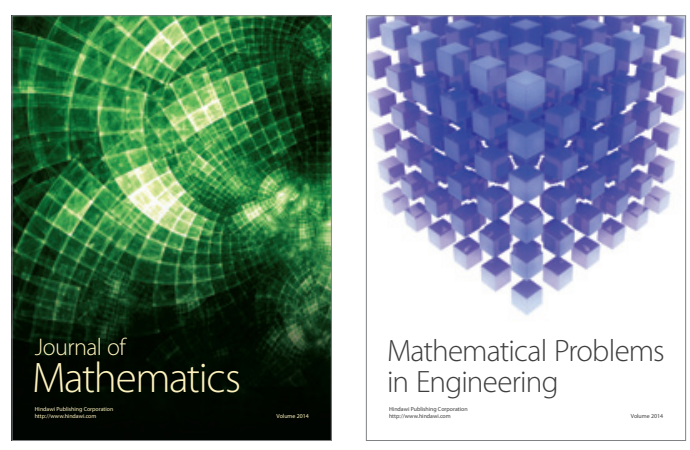

Mathematical Problems in Engineering
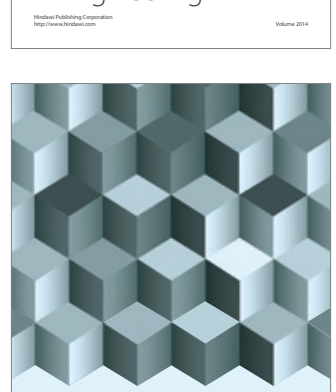

Journal of

Function Spaces
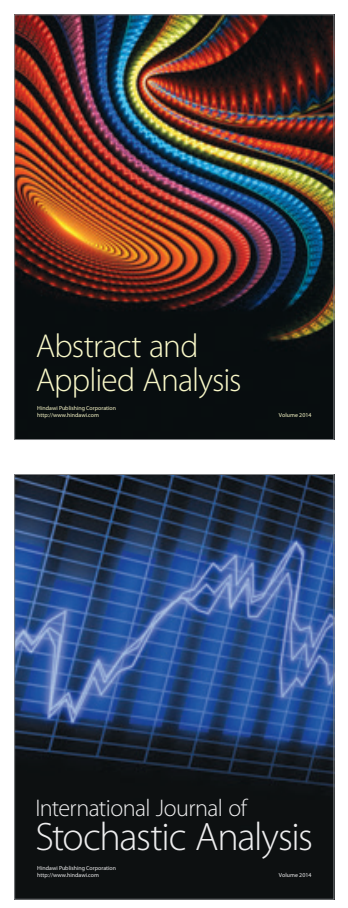

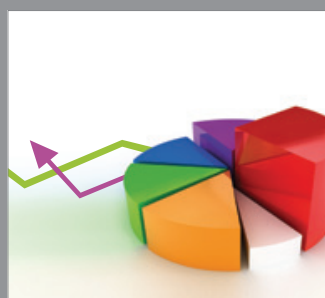

ournal of

Probability and Statistics

Promensencen
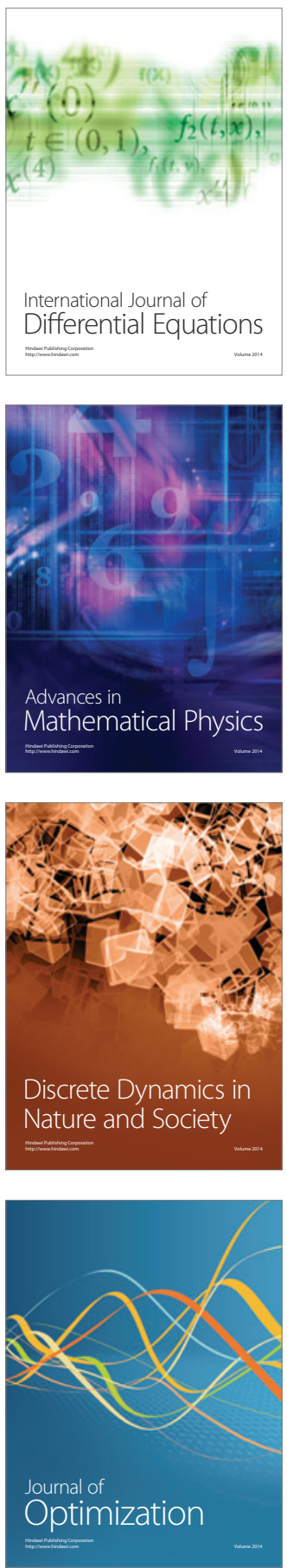